\title{
Recherche sur blé d'un paramètre de tolérance à Septoria nodorum : teneur des épis en acide abscissique
}

\author{
JF Bousquet 1, G Touraud 1, MT Piollat 1, U Bosch *, M Trottet 2 \\ ${ }^{1}$ INRA, station de pathologie végétale, laboratoire de physiopathologie, 78026 Versailles; \\ 2 INRA, station d'amélioration des plantes, 35650 Le Rheu, France
}

(Reçu le 13 mars 1991; accepté le 7 juillet 1991)

\begin{abstract}
Résumé - Cinq cultivars de blé d'hiver représentant un gradient de tolérance à la septoriose sont contaminés à 2 stades de leur développement. La concentration en acide abscissique (ABA) est déterminée dans les épis aux stades laiteux et pâteux du grain. Les symptômes sur feuilles et épis sont notés en cours de végétation. Le poids de 1000 grains est mesuré à la récolte. L'ABA s'accumule dans les épis septoriés proportionnellement aux symptômes observés. Le niveau de la relation entre les pertes de rendement et l'accumulation d'ABA dépend du stade de développement de la plante au moment de l'infection. L'utilisation de l'accumulation d'ABA en tant que marqueur de la tolérance est discutée.
\end{abstract}

Triticum aestivum = blé d'hiver / Phaerosphaeria nodorum / septoriose du blé / tolérance / sélection / rendement / symptôme / infection / stade de développement

Summary - Investigation for assessing the tolerance of winter wheat to Septoria nodorum : ABA content in ears. Five winter wheat varieties showing differences in tolerance to Septoria nodorum were inoculated at the stages of stem extension and early heading. Abscissic acid (ABA) content was determined in the ears at milky and mealy stages. Disease ratings were made on symptoms developing on ears and flag leaves and on the yield expressed in 1 000 -grain weight (table I). The infected ears accumulated ABA and this accumulation was highly correlated with ear symptoms (table II and fig 1). The level of the relationship between yield losses and ABA accumulation depends on the growth stage of the plant at the time of infection. When the infection took place at the stage of stem extension, there was a high correlation between yield losses and ABA accumulation in the ears of the latest maturing cultivars (fig 2). The earliest maturing cultivar (Talent) showed a significant accumulation of ABA from the milky stage but without correlation with yield losses. These observations suggest that there is a critical period during grain development and maturation. The use of $A B A$ accumulation as marker of tolerance is examined.

Triticum aestivum / Phaeosphaeria nodorum / glume blotch / tolerance / selection / grain yield / symptom / infection / growth stage 


\section{INTRODUCTION}

En diminuant le poids du grain, Septoria nodorum (forme téléomorphique : Phaeosphaeria nodorum) occasionne de sévères pertes de rendement (Baker, 1978). Actuellement, l'intensité des symptômes sur les parties aériennes de la plante et la diminution du poids de 1000 grains sont les 2 paramètres utilisés pour caractériser les génotypes. Cependant, l'analyse du comportement de plusieurs lignées fait apparaître que les pertes de rendement ne sont pas en corrélation avec l'intensité de l'infection (Brönnimann, 1968; Trottet et Merrien, 1982). Les lignées les moins affectées dans ce sens sont désignées comme tolérantes sensu Simons (Schafer, 1971). Des cas de résistance des tissus hôtes au développement du parasite sont décrits mais le niveau de la résistance est toujours partiel (King et al, 1983) et aucune lignée ne présente une résistance de haut niveau. Ainsi, la stratégie de la sélection du blé vis-à-vis de ce parasite consisterait surtout à caractériser des cas de tolérance (Brönnimann, 1968). Depuis les travaux de Brönnimann, l'identification de ce caractère se fait par l'analyse du poids de 1000 grains après inoculation massive par des spores de $S$ nodorum. Toutefois, ce paramètre correspond à une définition peu spécifique de la tolérance sensu Caldwell (Schafer, 1971) et les bases génétiques et physiologiques de la tolérance restent pratiquement inconnues (Baker, 1978). Un paramètre plus spécifique et plus précoce reste à définir. Les effets de la septoriose telles que la sénescence foliaire précoce (Verreet et al, 1987), la diminution de l'absorption du $\mathrm{CO}_{2}$ (Scharen et Taylor, 1968), l'inhibition de la germination et de la croissance de coléoptiles de blé (Babadoost et Hebert, 1984), ainsi que la plus faible accumulation de matière sèche dans les grains, suggèrent une intervention de l'ABA. De plus, l'implication de ce régulateur de croissance dans les maladies des plantes a déjà été rapportée (Pegg, 1976). Dans ces conditions, il paraît intéressant de suivre l'évolution de I'ABA dans les épis en relation avec les symptômes de la maladie (nécroses des enveloppes florales et diminution du poids de 1000 grains) et de rechercher, par l'examen de plusieurs cultivars, s'il existe une relation entre ce paramètre et le caractère de tolérance.

\section{MATÉRIEL ET MÉTHODES}

\section{Préparation du matériel végétal}

Cinq cultivars représentant un gradient de sensibilité à la septoriose sont utilisés. Les plantes sont cultivées en blocs randomisés sur des parcelles irriguées par aspersion, à la station d'amélioration des plantes de Rennes, dans les conditions précédemment décrites par Jahier et Trottet (1980). Les contaminations sont réalisées par pulvérisation à raison de $200 \mathrm{ml}$ par $\mathrm{m}^{2}$ d'une suspension de spores de $S$ nodorum renfermant $10^{6}$ spores par $\mathrm{ml}$. L'importance des pertes de rendement étant liée au stade de développement de la plante au moment de l'infection (Verreet et al, 1987), 2 modalités de contamination sont retenues. La première modalité est pratiquée à la montaison (stade GS 40-49 de l'échelle de Zadock et al, 1974). A ce stade les 5 variétés sont soumises à 2 contaminations à 3 semaines d'intervalle. La seconde modalité consiste en 1 contamination réalisée en début d'épiaison (stade GS 56-59) par pulvérisation de spores à une date unique tenant compte au mieux de la précocité de chaque variété établie comme il suit : Talent, Capitole, Rescler, Fidel et Arminda.

\section{Évaluation des symptômes}

L'intensité des nécroses sur feuilles et épis est exprimée en pourcentage de la surface totale de l'organe. La surface nécrosée de la dernière feuille est mesurée suivant la méthode de Jahier et Trottet (1980). Le nombre d'épillets infectés est évalué visuellement et reporté de façon linéaire selon une échelle allant de 0 (moins de $3 \%$ d'épillets infectés) à 4 (plus de $80 \%$ d'épillets infectés).

Le poids de matière sèche des épis est obtenu après dessiccation à l'étuve à $105^{\circ} \mathrm{C}$ pendant $2 \mathrm{j}$.

Le poids de 1000 grains est déterminé à la récolte après une période de rééquilibrage de l'humidité.

\section{Dosage de l'ABA}

Pour chaque variété, 10 épis sont prélevés sur le brin maître et les talles principales à 2 stades de développement du grain : stade laiteux (GS 73-79) et stade pâteux (GS 83-86). Les épis sont plongés sur place dans $200 \mathrm{ml}$ d'éthanol à $80 \%$ et stockés ainsi à l'obscurité, à $4^{\circ} \mathrm{C}$. Les dosages de l'ABA libre et de I'ABA lié sont effectués suivant la méthode décrite par Touraud et al (1986) avec quelques modifications mi- 
neures. Les échantillons sont broyés à l'aide d'un homogénéisateur Waring Blendor. Le broyat est filtré sur papier. L'extrait éthanolique est réduit par évaporation sous vide à $60^{\circ} \mathrm{C}$. Après ajustement du $\mathrm{pH}$ à 9 par de la soude $1 \mathrm{~N}$, cet extrait est fractionné 4 fois ( $/ \mathrm{v}$ ) contre de l'acétate d'éthyle. La fraction aqueuse, après acidification à $\mathrm{pH} 2,8$ par $\mathrm{HCl} 1 \mathrm{~N}$, est fractionnée 4 fois contre de l'éther éthylique $(\mathrm{v} / \mathrm{v})$. L'ABA lié contenu dans la fraction aqueuse est hydrolysé à $\mathrm{pH} 11$ pendant $1 \mathrm{~h}$ à $60^{\circ} \mathrm{C}$ puis fractionné à $\mathrm{pH} 2,8$ contre de l'éther éthylique. Les fractions contenant l'ABA libre ou I'ABA lié sont traitées de manière identique. Les fractions éthérées sont évaporées à sec et reprises par 3 $\mathrm{ml}$ de méthanol. Cet extrait est chromatographié sur couche mince de silice (Schleicher et Schüll, F1500, LS 254) par un mélange hexane/acétate d'éthyle/acide acétique $(60 / 40 / 5, v / v)$. Les zones correspondant à I'ABA, visualisées sous UV, sont éluées par $2,5 \mathrm{ml}$ d'acétate d'éthyle saturé d'eau. Après centrifugation, 1 $\mathrm{ml}$ de surnageant est évaporé sous vide et repris par $1,5 \mathrm{ml}$ de méthanol anhydre. Cet extrait est méthylé par le diazométhane, puis évaporé sous vide et le résidu repris par 1-2 $\mathrm{ml}$ de méthanol anhydre. Une partie aliquote de $1 \mu$ l est finalement injectée dans un chromatographe en phase gazeuse Girdel série 300 équipé d'un injecteur évaporateur type Ross, d'un détecteur à capture d'électrons et d'une colonne capillaire en silice fondue de $25 \mathrm{~m}$ de longueur et $0,32 \mathrm{~mm}$ de diamètre imprégnée de silicone fluoré type OV215. Les températures de travail sont respectivement de $205{ }^{\circ} \mathrm{C}$ pour la colonne, $250^{\circ} \mathrm{C}$ pour l'injecteur et $220^{\circ} \mathrm{C}$ pour le détecteur. Le débit du gaz vecteur (azote qualité $U$ ) au travers de la colonne est de $2 \mathrm{ml} /$ min. Dans ces conditions, les temps de rétention de
l'ABA sont de 7'10" pour l'isomère cis trans et 9'40" pour l'isomère trans trans. La plus faible quantité d'ABA détectable dans l'extrait final est d'environ $15.10^{-12} \mathrm{~g}$ par injection.

\section{RÉSULTATS}

\section{Influence du stade de développement sur l'importance de l'infection (tableau I)}

L'irrigation par aspersion a permis un très bon développement du $S$ nodorum et l'effet de la contamination est très significatif pour les 2 paramètres (nécroses et poids de 1000 grains).

Réalisée à la montaison, la contamination est peu destructrice du système foliaire et des différences, dans l'évolution des symptômes, peuvent être notées entre les cultivars. Par contre, pour une contamination se situant à l'épiaison, le pourcentage de surface nécrosée augmente très rapidement entre le 1 er juillet et le 4 juillet. En fait, ce symptôme est difficile à identifier en l'absence de pycnides et peut être confondu avec la sénescence naturelle.

Le classement variétal d'après le poids de 1000 grains souligne l'importance du stade physiologique de la plante au moment de l'infection.

Tableau I. Effets d'infection par $S$ nodorum, à 2 stades de développement du blé.

\begin{tabular}{|c|c|c|c|c|c|c|}
\hline \multirow[t]{3}{*}{ Variétés } & \multirow{3}{*}{$\begin{array}{l}\text { Dates de } \\
\text { contamination }\end{array}$} & \multicolumn{4}{|c|}{ Pourcentage de surface nécrosée } & \multirow{3}{*}{$\begin{array}{l}\text { Perte du poids } \\
\text { de } 1000 \text { grains } \\
\text { en } \% \text { du témoin }\end{array}$} \\
\hline & & & & surde & re feuille & \\
\hline & & 1 juillet & 4 juillet & 1 juillet & 4 juillet & \\
\hline \multirow[t]{3}{*}{ Talent } & Témoin & 0,0 & 0,0 & 0,2 & 1,0 & \\
\hline & Montaison & 0,2 & 0,2 & 1,0 & 2,7 & 3,5 \\
\hline & Epiaison & 10,0 & 10,0 & 12,5 & 80,0 & 23,8 \\
\hline \multirow[t]{3}{*}{ Capitole } & Témoin & 0,0 & 0,2 & 1,0 & 2,0 & \\
\hline & Montaison & 0,0 & 0,2 & 10,0 & 18,7 & 13,3 \\
\hline & Epiaison & 4,2 & 10,0 & 32,5 & 80,0 & 17,0 \\
\hline \multirow[t]{3}{*}{ Rescler } & Témoin & 0,0 & 0,0 & 1,0 & 1,0 & \\
\hline & Montaison & 0,0 & 0,2 & 2,0 & 2,0 & 8,0 \\
\hline & Epiaison & 2,7 & 5,5 & 27,5 & 65,0 & 18,4 \\
\hline \multirow[t]{3}{*}{ Fidel } & Témoin & 0,2 & 0,2 & 0,6 & 5,5 & \\
\hline & Montaison & 0,2 & 0,2 & 1,0 & 16,2 & 6,6 \\
\hline & Epiaison & 3,0 & 5,0 & 3,0 & 45,0 & 17,4 \\
\hline \multirow[t]{3}{*}{ Arminda } & Témoin & 0,0 & 0,0 & 0,2 & 0,2 & \\
\hline & Montaison & 0,0 & 0,2 & 0,2 & 0,2 & 3,7 \\
\hline & Epiaison & 1,0 & 10,0 & 2,0 & 30,0 & 30,7 \\
\hline
\end{tabular}


Les pertes observées sont en accord avec celles obtenues durant plusieurs années dans des conditions expérimentales similaires (Trottet et al, 1975; Trottet et Merrien, 1982).

Pour l'ensemble des variétés, le fait le plus remarquable est la faible corrélation entre les symptômes sur la dernière feuille ou l'épi et les pertes de rendement dans le cas d'une contamination à l'épiaison ( $r$ compris entre 0,45 et 0,73 ). Toutefois, un coefficient de corrélation élevé $(r=$ 0,93 ) est observé uniquement pour les symptômes sur feuille dans le cas d'une contamination à la montaison.

Une analyse détaillée montre que les symptômes sur épi et dernière feuille sont plus marqués pour les variétés Talent et Capitole bien que les pertes sur le poids de 1000 grains restent limitées. Aussi pouvons-nous considérer ces 2 variétés comme tolérantes. À l'opposé, la variété Arminda présente des symptômes réduits sur feuilles et sur épi mais exprime des pertes en poids de 1000 grains élevées. Enfin, Rescler et Fidel ont un comportement de type intermédiaire. Bien que ce classement puisse dépendre de la précocité des variétés (Rosielle et Brown, 1980), un tel échantillonnage offre une échelle de comportement permettant de rechercher une éventuelle relation entre les symptômes ou le poids de 1000 grains d'une part, et l'accumulation de l'ABA d'autre part.

\section{Évolution de l'ABA dans les épis (tableau II)}

Pour les plantes témoins, le taux d'ABA dans les épis augmente de façon très significative entre le stade laiteux et le stade pâteux.

Pour toutes les variétés à l'exception de Talent, l'infection ne provoque pas au stade laiteux une augmentation notable du taux d'ABA. Les prélèvements faits au stade pâteux montrent une augmentation s'échelonnant de 21 à $74 \%$ par rapport aux témoins sains. Cette accumulation d'ABA plus forte pour une contamination à l'épiaison que pour une contamination à la montaison apparaît en ce sens représentative de la maladie.

Chez Talent, on observe dans les épis contaminés, dès le stade laiteux, une quantité totale d'ABA 2 fois plus importante que dans les épis sains. Cette donnée serait à rattacher à la grande précocité de cette variété (la plus précoce des 5).
Le pourcentage de l'ABA lié par rapport à l'ABA total est toujours réduit et décroît faiblement ou même pas du tout avec l'infection. Ces faits suggèrent que l'ABA total s'accumulant en réponse à l'infection serait synthétisé de novo.

\section{Relation entre les symptômes sur épi et l'accumulation de l'ABA (fig 1)}

Pour l'ensemble des échantillons, l'accumulation de I'ABA présente une corrélation linéaire avec les symptômes visuels sur épi. Le coefficient de corrélation est élevé $(r=0,91)$ malgré la difficulté d'appréciation des symptômes dans les classes intermédiaires.

\section{Relation entre les pertes sur le poids de 1000 grains et l'accumulation de l'ABA (fig 2)}

Les contaminations aux stades montaison et épiaison n'influencent pas de la même façon ces 2 paramètres. Pour les contaminations au stade montaison et si l'on excepte le cultivar Talent, il existe au stade pâteux une corrélation entre les pertes de rendement et l'accumulation d'ABA (significatif à $5 \%$ ). Au stade laiteux, $r$ est égal à 0,89 et non significatif. Dans le cas de contaminations à l'épiaison, l'absence de corrélation

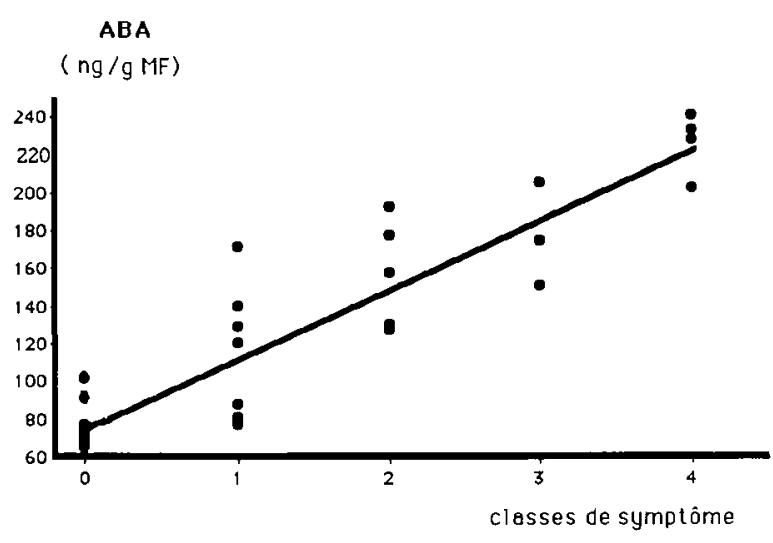

Fig 1. Analyse de la relation entre les symptômes visuels sur épis et leur teneur en ABA total. Les épis sont récoltés sur des plantes saines et contaminées aux stades montaison el épiaison. Les valeurs prises en considération concernent les variétés Talent, Capitole, Rescler et Fidel. Chaque point correspond à un échantillon de 10 épis. La droite de régression est $y=37,2 x+74,7 ; r=0,91$. 
Septoriose du blé : ABA et tolérance

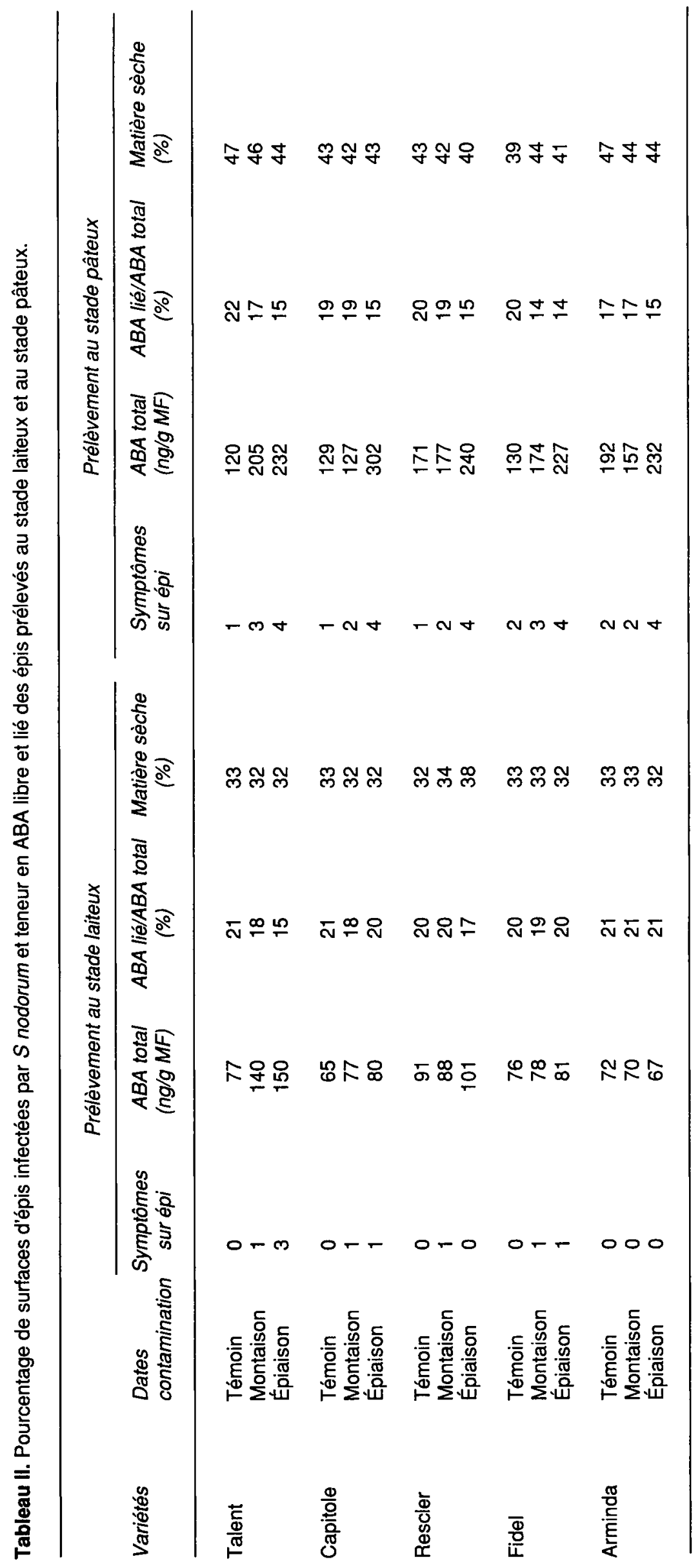




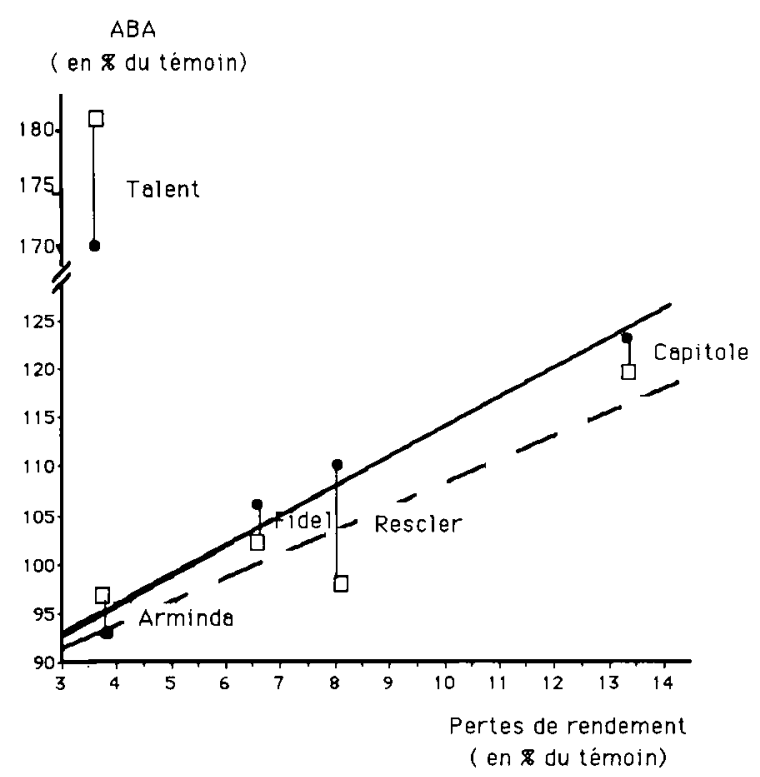

Fig 2. Relation entre le pourcentage de pertes noté en poids de 1000 grains et la teneur en ABA. Les plantes sont infectées à la montaison. $\square$, au stade laiteux; la ligne discontinue représente la droite de régression $(y=2,2 x+86,2 ; r=0,89)$; $\bullet$, au stade pâteux; la ligne continue représente la droite de régression $(y=3,0 x+84,1 ; r=0,97)$. Les données de la variété Talent n'ont pas été prises en considération pour le calcul des droites de régression.

entre teneur en $A B A$ et pourcentage de perte est observée et discutée ultérieurement.

\section{CONCLUSION ET DISCUSSION}

\section{Effet de la septoriose sur l'accumulation d'ABA}

Comme des expériences antérieures l'avaient indiqué (Bousquet et al, 1990), la septoriose provoque une accumulation accrue d'ABA dans les épis. Cette augmentation est proportionnelle aux dégâts visuels observés sur les échantillons analysés. Or l'ABA n'induit pas la formation de nécroses (revue de Hirai, 1986). Si l'observation est applicable à ce modèle expérimental, les nécroses observées ne seraient pas imputables à I'ABA accumulé mais à la production de toxines ou d'enzymes par le parasite (Kent et Strobel, 1976; Jeger et al, 1983; Magro, 1984). Par contre la corrélation entre l'accumulation d'ABA et le pourcentage de surfaces nécrosées suggère que cette accumulation est une conséquence de l'activité du champignon.

\section{Modalités d'accumulation de l'ABA}

L'ABA lié ne représente que le cinquième de I'ABA total. Sa quantité décroît faiblement avec l'infection. L'ABA dont on observe l'accumulation serait donc synthétisé de novo en réponse à l'infection. Dewdney et McWha (1978) formulent l'hypothèse que, malgré le transport de l'ABA dans la plante, l'accumulation durant le développement du grain résulte essentiellement d'une biosynthèse in situ dans le grain. Bronnimann (1968) suggère que des substances toxiques libérées par le parasite seraient en partie responsables de l'échaudage du grain. De ce fait cette biosynthèse se ferait sous l'influence de substances diffusibles excrétées par le champignon.

En 1968, Scharen et Taylor ont montré que l'assimilation nette du $\mathrm{CO}_{2}$ diminue lors d'une infection par $S$ nodorum et que les pertes de rendement sont en corrélation avec cette diminution de la photosynthèse. Plus récemment, Rooney (1989) a confirmé que la production de la biomasse et les échanges gazeux chez le blé d'hiver sont réduits par l'inoculation de la dernière feuille avec $S$ nodorum. Selon Van Der Wall et Cowan (1974), l'infection diminue aussi la transpiration.

$S$ nodorum produit diverses isocoumarines dont la melléine (ou ochracine) (Bousquet et Skajennikoff, 1974). Ce composé augmente la résistance stomatique et diminue l'assimilation du $\mathrm{CO}_{2}$ lorsqu'il est apporté par voie racinaire à des plantules de blé (Bousquet et al, 1977). Toutefois une action directe de la melléine sur les stomates n'est pas observée (Laffray et al, 1982; Bethenod et al, 1982). La question est de savoir si l'action de la melléine sur la fixation du carbone est directe ou passe par une voie impliquant la production d'ABA dont l'une des activités biologiques est sa capacité à causer la fermeture des stomates et à réduire la transpiration (Hirai, 1986).

\section{Effet de l'accumulation d'ABA sur le remplissage du grain}

Comparée à l'évolution de l'ABA dans les épis sains entre les stades laiteux et pâteux, l'augmentation observée peut avoir une signification physiologique. En effet, pendant cette période de maturation du grain, la quantité d'ABA dans l'épi sain augmente jusqu'à 2 fois (King, 1976) alors 
que le contenu en ABA des épis infectés est multiplié par 4. Or l'ABA joue un rôle essentiel dans le contrôle de la durée de remplissage du grain (Michael et Beringer, 1980). L'échaudage lié à la septoriose trouverait son origine dans cette accumulation.

L'importance des pertes occasionnées par la septoriose est déterminée par le stade de développement auquel la plante est attaquée (Verreet et al, 1987). L'effet le plus néfaste est observé pour une infection se situant entre l'émergence de l'épi et la floraison (King et al, 1983). Nos résultats confirment cette observation. Pour une infection pratiquée à la montaison, les pertes de rendement restent limitées (3-14\%) et sont en étroite corrélation avec l'accumulation d'ABA dans le cas de 4 des 5 variétés étudiées. Plus précoce, la cinquième variété (Talent) présente un comportement différent. Quand la contamination a lieu plus tardivement, les pertes de rendement sont plus élevées (17-31\%). Ces résultats suggèrent qu'à partir d'un certain stade de développement de l'épi, correspondant à l'épiaison, la précarité de certaines fonctions physiologiques exacerbe les perturbations causées par le parasite. Ce fait confirme que les attaques les plus efficaces sont celles qui affectent l'épi durant la phase correspondant au maximum de teneur en eau de l'épi, ou palier de poids d'eau (Doussinault et al, 1972). Cette période est critique pour le développement du grain de blé. Tout incident perturbant la physiologie de la plante à ce stade a des conséquences importantes sur l'accumulation de la matière sèche (Trottet et al, 1975). Une contamination, à ce stade physiologique, cause une importante accumulation d'ABA pour les 5 variétés choisies, mais cette augmentation est sans relation avec les pertes de rendement exprimées en poids de 1000 grains. S'il est vrai que le poids de 1000 grains est une composante du rendement très affectée par l'infection (Wafford et Whitbread, 1978), le nombre de grains par plante ou par épi est également et largement réduit par la maladie (Scharen et Taylor, 1968; Brönnimann, Sally et Sharp, 1972; Scott, 1973). La perte de rendement en pourcentage de poids de 1000 grains ne peut être prise comme unique paramètre car, malgré une photosynthèse nette réduite ou un transfert limité des réserves carbonées, le poids d'un grain peut rester relativement constant du fait d'une réduction du nombre de grains par épi (Triboï, comm pers). Le poids d'un grain d'un génotype ayant ce type de réponse à l'infection sera stable et le potentiel productif de ce génotype sera plus faible. Les composantes du rendement telles que nombre de grains par épi et structure du peuplement devront donc être prises en considération.

Les symptômes visibles sur la dernière feuille entre la fin du stade aqueux et la fin du stade laiteux expliquent la diminution du poids de 1000 grains au niveau de $30 \%$ (Bronnimann, 1968; Trottet et Merrien, 1982). La baisse de l'assimilation du $\mathrm{CO}_{2}$ par les plantes septoriées fut proposée comme critère précoce pour sélectionner des génotypes tolérants aux effets de la septoriose (Scharen et Krupinsky, 1969). De plus, des différences génotypiques dans les concentrations d'ABA ont été observées chez des blés de printemps soumis à un stress hydrique (Zeevaart et Creelman, 1988). Dans la pratique et sur la base de cette expérimentation, les différences de précocité entre les variétés rendent difficile la mise en évidence d'une relation entre les pertes de rendement et l'accumulation d'ABA. Pour un génotype qui présente un grand nombre de grains par épi et un tallage important, le remplissage du grain s'effectue essentiellement à partir de la photosynthèse après floraison. Sa vitesse est plus faible mais sa durée plus longue. Un génotype ayant ces caractéristiques risque d'être plus touché par une telle maladie. Arminda pourrait rentrer dans cette catégorie (Triboï, 1990). En conséquence, si l'accumulation de l'ABA peut être retenue comme un indicateur de réponse du blé à l'infection, il semble difficile de considérer ce paramètre comme unique marqueur de différences génotypiques dans la tolérance sensu Brönnimann (1974) à un même niveau d'attaque par $S$ nodorum. Trottet et Merrien (1982) ont analysé l'effet de la septoriose sur le poids de 50 épis, le poids des grains dans 50 épis, le poids de 1000 grains et le nombre de grains par épi. L'effet de l'inoculation est significatif pour les 3 premiers paramètres et une interaction lignées $x$ traitements (non inoculation et inoculation) significative est observée pour les 4 . Ces auteurs concluent à un effet spécifique de la septoriose sur les facteurs du rendement, chaque variété présentant un comportement différencié. 


\section{REMERCIEMENTS}

Cette recherche a été supportée par un contrat INRAONIC-ITCF à JF Bousquet et M Trottet et une bourse du DADD à U Bosch. Les auteurs remercient le Dr Triboï de la station d'agronomie de Clermont-Ferrand (INRA) pour la lecture extrêmement constructive du manuscrit et Madame Fiala (Département de Pathologie Végétale, INRA, Versailles) pour son aide à réaliser la bibliographie.

\section{RÉFÉRENCES}

Babadoost M, Hébert TT (1984) Incidence of Septoria nodorum in wheat seed and its effects on plant growth and grain yield. Plant Dis 68, 125-129

Baker EA (1978) Septoria- the lurking threat to wheat yields. EPPO Bull 8, 9-20

Bethenod $O$, Bousquet JF, Laffray D, Louguet $P$ (1982) Réexamen des modalités d'action de l'ochracine sur la conductance stomatique des feuilles de plantules de blé, Triticum aestivum L, cv Étoile de Choisy. Agronomie 2, 99-102

Bousquet JF, Skajennikoff M (1974) Isolement et mode d'action d'une phytotoxine produite en culture par Septoria nodorum Berk. Phytopathol Z 80, 355360

Bousquet JF, Skajennikoff M, Bethenod O, Chartier $P$ (1977) Effets dépressifs de l'ochracine, métabolite produit par Septoria nodorum sur l'assimilation nette de plantules de Blé. Ann Phytopathol 93, 503510

Bousquet JF, Touraud G, Piollat MT, Bosch U, Trottet $M$ (1990) ABA accumulation in wheat heads inoculated with Septoria nodorum in the field conditions. J Agron Crop Sci 165, 297-300

Brönnimann A (1968) Zur Kenntniss von Septoria nodorum Berk dem erreger der Spelzenbräune und einer Blattdürre des Weizens. Phytopathol Z 61, 101-146

Brönnimann A (1974) Bisherige Erfahrungen in der Züchtung auf Septoria-Toleranz (Septoria nodorum Berk) bei Weizen. Schweiz Landwirtsch Forsch 13, 491-500

Brönnimann A, Sally BK, Sharp EL (1972) Investigations on Septoria nodorum in spring wheat in Montana. Plant Dis Rep 56, 188-191

Dewdney Sj, McWha JA (1978) The metabolism and transport of abscissic acid during grain fill in wheat. $J$ Exp Bot 29, 1299-1308

Doussinault G, Boixiere R, Chaussee D (1972) Incidence des attaques de Septoria nodorum et $S$ tritici sur l'accumulation de matière sèche dans le grain de deux variétés de blé tendre. Sci Agron Rennes, $10 \mathrm{p}$

Hirai N (1986) Absisic acid. In : Chemistry of Plant Hormones (N Takahashi, ed) CRC Press

Jahier J, Trottet M (1980) Conséquences of an attack of Septoria nodorum on the accumulation of dry matter in the grain of Aegilops squarrosa. Cereal Res Commun 8, 325-330

Jeger MJ, Jones DG, Griffiths E (1983) Components of partial resistance of wheat seedlings to Septoria nodorum. Euphytica 32, 575-584

Kent SS, Strobel GA (1976) Phytotoxin from Septoria nodorum. Trans Br Mycol Soc 67, 354-358

King RW (1976) Abscissic acid in developing wheat grains and its relationship to grain growth and maturation. Planta 132, 43-51

King JE, Cook RJ, Melville SC (1983) A review of Septoria diseases of wheat and barley. Ann Appl Biol $103,345-373$

Laffray D, Bousquet JF, Bethenod O, Louguet P (1982) Mécanismes de l'action dépressive de l'ochracine, phytotoxine synthétisée par Septoria nodorum Berk sur le degré d'ouverture des stomates des feuilles de plantules de blé et de Pelargonium $\times$ hortorum. Agronomie 2, 25-30

Magro $P$ (1984) Production of polysaccharidedegrading enzymes by Septoria nodorum in culture and during pathogenesis. Plant Sci Lett 37, 63-68

Michael G, Beringer H (1980) The role of hormones in yield formation. In: Physiological aspects of crop productivity, Proc 15th Coll, Wageningen, 85-116

Pegg GF (1976) Endogenous inhibitors in healthy and disease plants. In : Encyclopedia of Plant Physiology, New Series vol 4, Physiological Plant Pathology (R Heitefuss, PH Williams, eds) 607-616

Rooney JM (1989) Biomass production and gas exchange of winter wheat as affected by flag leaf inoculations with Septoria nodorum. New Phytol 112, 229-234

Rosielle AA, Brown AGP (1980) Selection for resistance to Septoria nodorum on wheat cultivars. Ann Appl Biol 75, 321-329

Schafer JF (1971) Tolerance to plant disease. Annu Rev Phytopathol 9, 235-252

Scharen AL, Taylor JM (1968) $\mathrm{CO}_{2}$ assimilation and yield of Little Club wheat infected by Septoria nodorum. Phytopathology 58, 447-451

Scharen AL, Krupinsky JM (1969) Effet of Septoria nodorum infection on $\mathrm{CO}_{2}$ absorption and yield of wheat. Phytopathology 59, 1298-1301

Scott PR (1973) Incidence and effets of Septoria nodorum on wheat cultivars. Ann Appl Biol 75, 321 329

Touraud G, Leydecker MT, Darmency H (1986) Abscisic acid in triazine-resistant and susceptible $P o a$ annua. Plant Sci 49, 81-83

Triboi E (1990) Modèle d'élaboration du poids du grain chez le blé tendre (Triticum aestivum em Thell). Agronomie 10, 191-200

Trottet M, Merrien P (1982) Analyse du comportement du vingt lignées de blé tendre vis-à-vis de Septoria nodorum Berk. Agronomie 2, 727-734

Trottet M, Dosba F, Doussinault G (1975) Conséquences d'une attaque de Septoria nodorum (Berk) 
sur la formation du grain chez Triticum aestivum (L) et chez Aegilops ventricosa (Tausch). Incidences en sélection. Ann Amelior Plant 25, 265-276

Van der Wall AF, Cowan MC (1974) An ecophysiological approach to crop losses exemplified in the system wheat, leaf rust and glume blotch II. Development, growth and transpiration of uninfected plants and plants infected with Puccinia recondita f $\mathrm{sp}$ triticina and/or Septoria nodorum in climate chamber experiment. Neth J Plant Pathol 80, 192-214

Verreet JA, Hoffmann GM, Amberger A (1987) Auswirkungen der Infektion durch Septoria nodorum in verschiedenen Entwicklungsstadien des Weizens auf die Produktionsleistung. J Plant Dis Prot 94, 283-300

Wafford JD, Whitbread R (1978) Effects of inoculation with Septoria nodorum on yield components of spring wheat. Ann App/ Bio/ 90, 323-328

Zadock JC, Chang TT, Konzak CF (1974) A decimal code for the growth stages of cereals. Weed Res $14,415-421$

Zeevaart JAD, Creelman RA (1988) Metabolism and physiology of abscisic acid. Ann Rev Plant Physiol Plant Mol Biol 39, 439-473 